\title{
Turning off quantum duality
}

\author{
X.-F. Qian ${ }^{1},{ }^{1,2,3,{ }^{*}}$ K. Konthasinghe, ${ }^{1,2}$ S. K. Manikandan, ${ }^{1,4}$ D. Spiecker, ${ }^{5}$ A. N. Vamivakas, ${ }^{1,2,4}$ and J. H. Eberly ${ }^{1,2,4}$ \\ ${ }^{1}$ Center for Coherence and Quantum Optics, University of Rochester, Rochester, New York 14627, USA \\ ${ }^{2}$ The Institute of Optics, University of Rochester, Rochester, New York 14627, USA \\ ${ }^{3}$ Department of Physics, Stevens Institute of Technology, Hoboken, New Jersey 07030, USA \\ ${ }^{4}$ Department of Physics \& Astronomy, University of Rochester, Rochester, New York 14627, USA \\ ${ }^{5}$ National Technical Institute for the Deaf, Science and Mathematics Department, \\ Rochester Institute of Technology, Rochester, New York 14623, USA
}

(Received 5 March 2019; published 15 January 2020)

\begin{abstract}
We provide the first experimental confirmation of a three-way quantum coherence identity possessed by single pure-state photons. Our experimental results demonstrate that traditional wave-particle duality is specifically limited by this identity. As a consequence, we show that quantum duality itself can be amplified, attenuated, or turned completely off. In the Young double-slit context this quantum coherence identity is found to be directly relevant, and it supplies a rare quantitative backup for one of Bohr's philosophical pronouncements.
\end{abstract}

DOI: 10.1103/PhysRevResearch.2.012016

Introduction. de Broglie's hypothesis of quantum duality, presented in his 1924 doctoral thesis [1], asserted that every quantum-mechanical entity will act as both a particle and a wave, two aspects equally real and contradictory. More than 90 years later the duality hypothesis, ridiculous in ordinary discourse, is accepted by physicists as truly inescapable.

Feynman famously celebrated quantum duality “.... as] a phenomenon which is impossible, absolutely impossible, to explain in any classical way, and which has in it the heart of quantum mechanics. In reality it contains the only mystery." [2]. However, the mystery can be quantified, which means that duality can be tested.

Remarkably, this has never been done at the single-particle level required to test de Broglie's duality. For any experiment analogous to Young-type two-beam interference [3] (suggested in Fig. 1), the work of Wootters and Zurek in 1979 [4] introduced the standard route for analysis and eliminated the tendency to consider duality as an exclusive either-or wave-particle restriction. They showed that it is better seen as a combination in which both features can be simultaneously active: $V$ for visibility of interference fringes measuring waveness and $D$ for separate light path distinctiveness measuring particle probability. In several ways [5-7] these were then shown to be quantified in the inequality

$$
V^{2}+D^{2} \leqslant 1
$$

Without testing duality itself, this quantification has been repeatedly exhibited and employed in various quantum sce-

\footnotetext{
*xiaofeng.qian@stevens.edu

Published by the American Physical Society under the terms of the Creative Commons Attribution 4.0 International license. Further distribution of this work must maintain attribution to the author(s) and the published article's title, journal citation, and DOI.
}

narios [8,9]. Examples are atom interferometry, path and phase relations of photonic orbital angular momenta, and quantum delayed-choice experiments [10-19]. The full range of both $V$ and $D$ between zero and unity has been explored, but when reporting single-particle experiments only in the specially restricted limit case $V^{2}+D^{2}=1$. No single-particle experiment has tested either the inequality (1) or the singleparticle duality more generally.

One must remember that there is another mystery that is as deeply embedded within quantum theory as duality. This is entanglement, known for its mysterious hands-off control of Schrödinger's hypothetical cat. A recent classical optical analysis by three of us [20] (QVE for short) suggested that entanglement is a coherence missing from Eq. (1). This does not contradict the Wootters-Zurek finding. Concurrence $C$ [21] is recognized as the appropriate entanglement measure in a qubitlike (two-beam) interference context, and the QVE analysis suggests that concurrence enters single-particle

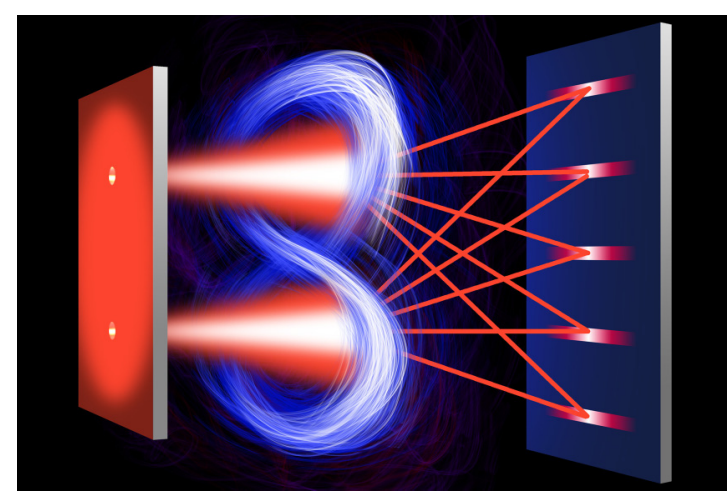

FIG. 1. Artistic conception of a generic Young-type experimental setup suggesting how entanglement engages beam distinguishability and interference visibility, all being necessarily interrelated. The source screen with the two pinholes is illuminated from the left by photons arriving singly. 
duality directly because it completes the inequality (1) to an equality, a quadratic identity for two-beam interference:

$$
V^{2}+D^{2}+C^{2}=1 .
$$

By convention (see Feynman [2]), duality refers to properties of a single physical entity. This is also our focus here, although we point out that a similar equality has been derived for the case of two qubits [22]. Our report is about the first experimental observations that test the quantification of duality. Our test is compatible with the presence of perfectly coherent single-particle entanglement via concurrence. Moreover, we explain in our concluding remarks that identity (2) allows us a rare experimental confirmation. We provide quantitative support for one of the many semiphilosophical pronouncements that Bohr was known for and which are almost never open to direct test.

The prior QVE analysis is not fully compelling because it was based on classical optical physics, and the reported experiments relied on entirely nonquantum instrumentation and detection. However, the classical optical derivation did take advantage of a mathematical inseparability. As Schrödinger noted when he introduced the term "entanglement" to quantum theory [24], such inseparability is the signal of entanglement. This is true whether the states being entangled are vectors in quantum Hilbert space or in classical linear function space. Thus the question is, does the three-part classical identity (2) derived in QVE also apply to individual quantum particles? In the following paragraphs we present the results of fully quantum single-particle (photon) self-interference experiments that give a positive answer to the question and thus represent the first completely tested quantification of quantum duality.

Experimental background. Here we present the results of our experiments, made with individual pure-state photons that create Young-type two-beam, or two-way Mach-Zehnder, self-interference. To begin the quantum experimental discussion, we repeat that de Broglie's duality is understood as an inescapable part of the nature of every single electron, photon, neutron, atom, etc. Thus, in the following we stick to the original discussions of single-particle duality and do not consider coherences of multiple particles (see, e.g., Refs. [22,23]).

The details of a two-beam interference experiment (recall Wootters and Zurek [4]) can be arranged for a given input photon pure state to yield any value of $V$ and any value of $D$ consistent with the often-proved inequality $V^{2}+D^{2} \leqslant 1$. Obviously, when the single photon is completely a particle $(D=1)$ then there should be no wave character $(V=0)$, and vice versa. However, relation (1) does not really predict a balanced exchange between $V$ and $D$ simply because the inequality permits a decrease of $D$ and $V$ together, or an increase by both. It even allows the extreme decrease $V=$ $D=0$ to exist (neither wave nor particle-see Sperling et al. [25]) while there is still a photon on hand. Such a photon cannot be nothing. One sees that something must be missing from $V^{2}+D^{2} \leqslant 1$.

Our task is to examine experimentally the proposition that $V$ and $D$ are restricted in a way that the inequality does not address. That is, the QVE condition $V^{2}+D^{2}+C^{2}=$ 1 is proposed as the correct general single-particle duality restriction. To proceed, the generic single-photon pure state to be tested is given by

$$
|\Psi\rangle=c_{a}\left|\mathbb{1}_{a}\right\rangle \otimes\left|\phi_{a}\right\rangle+c_{b}\left|\mathbb{1}_{b}\right\rangle \otimes\left|\phi_{b}\right\rangle
$$

where $c_{a}$ and $c_{b}$ are normalized coefficients with $\left|c_{a}\right|^{2}+$ $\left|c_{b}\right|^{2}=1$, and $\left|\mathbb{1}_{a}\right\rangle$ and $\left|\mathbb{1}_{b}\right\rangle$ are single-photon mode states indicating respectively one photon in propagating modes $a$ and $b$ and no photon elsewhere. Here $\left|\phi_{a}\right\rangle$ and $\left|\phi_{b}\right\rangle$ are two corresponding normalized states of all the remaining intrinsic (usually continuous) degrees of freedom (e.g., polarization, temporal mode, etc.) of the single photon with correlation defined as $|\gamma|=\left|\left\langle\phi_{a} \mid \phi_{b}\right\rangle\right| \leqslant 1$. From Eq. (3) and the expression for $\gamma$, one can quickly retrieve inequality (1) in the form

$$
V^{2}+D^{2}=1-4\left|c_{a}^{*} c_{b}\right|^{2}\left(1-|\gamma|^{2}\right) \leqslant 1,
$$

which our experiments show to be incomplete.

Single-photon generation. We employ a defect-hosted monolayer hexagonal boron nitride $(\mathrm{hBN})$ quantum emitter to serve as a single-photon source. Studies of a two-dimensional hBN quantum emitter as a quantum light source were started in 2015 by Tran et al. [26]. In this Rapid Communication we make the first systematic use of such hBN-generated photons in the realization and measurement of self-interference and single-photon entanglement. Our two-dimensional hBN sample resides on a silicon substrate $(\approx 5 \mathrm{~mm} \times 5 \mathrm{~mm}$ in size $)$ which is mounted (on an X-Y-Z Piezo) in an Attocube cryostat maintained at $10 \mathrm{~K}$. A Ti:sapphire laser operated at $725 \mathrm{~nm}$ is directed to the sample through a confocal microscope to produce off-resonant excitations of an hBN quantum dot. The emission spectrum, illustrated in Fig. 2(a), shows a bright zero-phonon line centered at $796.03 \mathrm{~nm}$ with a linewidth of $\leqslant 0.2 \mathrm{~nm}$ (limited by the instrument resolution $0.2 \mathrm{~nm}$ ). The narrow linewidth promises good coherence with singlephoton interference visibility up to $98.3 \%$ realized in the measurement stage. The second-order correlation function $g^{(2)}(\tau)$ of the detected signal is measured through a Brown-Twiss setup. Due to the detection resolution limit, the minimum value measured is $g^{(2)}(0)=0.29$, indicating single-photon nature, as shown in Fig. 2(b). The detailed properties of the hBN photon source are described in Ref. [27].

Single-photon entangled state preparation. We prepared seven different single-photon states based on Eq. (3), for testing the completed three-way QVE identity $V^{2}+D^{2}+C^{2}=$ 1. We employed a Mach-Zehnder interferometer, an analog of Young's double slits, to create the path states $\left|\mathbb{1}_{a}\right\rangle$ and $\left|\mathbb{1}_{b}\right\rangle$. For practical consideration and without loss of generality, the states of all remaining degrees of freedom of the photons $\left|\phi_{a}\right\rangle$ and $\left|\phi_{b}\right\rangle$, are represented by the polarization states $\left|s_{a}\right\rangle$ and $\left|s_{b}\right\rangle$.

The hBN-quantum-dot-emitted single photon is spectrally filtered with a $10-\mathrm{nm}$ bandpass filter and polarization oriented with a horizontal polarizer $|h\rangle$. As shown in Fig. 2(c), the polarized single photon passes through a half-wave plate (HWP1) and changes into an arbitrarily polarized state $|s\rangle$. A polarizing beam splitter (PBS) separates the horizontal $|h\rangle$ and vertical $|v\rangle$ polarizations into paths $a$ and $b$, respectively. The transmitted component, $\left|\mathbb{1}_{a}\right\rangle \otimes|h\rangle$, in path $a$ passes through a $50 / 50$ beamsplitter (BS1) that directs to a mirror mounted on a translation stage, before heading to the second $50 / 50$ beam splitter (BS2). The reflected component of the PBS in path $b$ passes through a half-wave plate (HWP2) to 

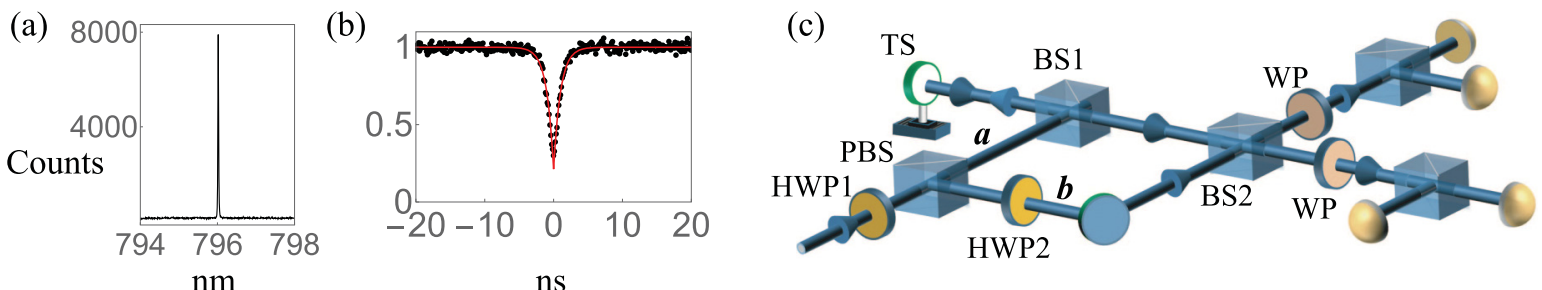

FIG. 2. Experimental aspects in testing the three-part single-particle duality identity $V^{2}+D^{2}+C^{2}=1$ with hBN-generated individual entangled pure-state photons defined by Eq. (5). Details of panels (a)-(c) are described in the text.

become $\left|\mathbb{1}_{b}\right\rangle \otimes\left|s_{b}\right\rangle$, where $\left|s_{b}\right\rangle=e^{i \xi} \cos \theta|h\rangle+\sin \theta|v\rangle$. Then the single-photon state entering the combining beam splitter (BS2) can be described as

$$
|\Psi\rangle=c_{a}\left|\mathbb{1}_{a}\right\rangle \otimes\left|s_{a}\right\rangle+c_{b}\left|\mathbb{1}_{b}\right\rangle \otimes\left|s_{b}\right\rangle,
$$

with $\left|s_{a}\right\rangle=|h\rangle$ representing horizontal polarization. Here the amplitude ratio $R=\left|c_{b} / c_{a}\right|$ of the two components is controlled by HWP1. The overlap of the two polarization states can be characterized as $\gamma=\left\langle s_{a} \mid s_{b}\right\rangle=\cos \theta e^{i \xi}$, where $\theta$ is controlled by HWP 2 in path $b$ and $\xi$ is manipulated by the translation stage in path $a$.

According to the quadratic QVE identity (2), the values of $(V, D, C)$ occupy the positive octant of the $V D C$ sphere. We chose seven representative sets of $(V, D, C)$ distributed over this octant surface. These target sets are determined respectively by the nodes of six grid lines on the $V D C$ sphere, i.e., $V=0, D=0, C=0, V / D=1, V / C=1$, and $C / D=1$, as shown in Fig. 3. The detailed values of all sets of $(V, D, C)$ are given in detail in Table I.

We achieved these target $(V, D, C)$ values by generating corresponding single-photon states as defined in Eq. (5), by way of the state control parameters $R$ and $\theta$. This is based on the fact that visibility, distinguishability, and entanglement

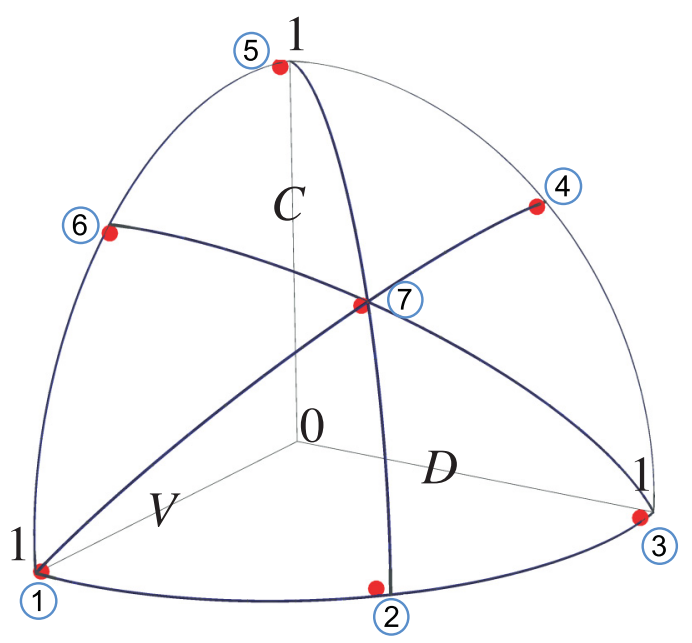

FIG. 3. An octant is shown of the $V D C$ sphere defined by the QVE duality identity $V^{2}+D^{2}+C^{2}=1$. Our experiments subject a generic single-photon pure state to a variety of different two-beam interference experiments. These yield a wide range of $V$ and $D$ values. The observed values of $V^{2}+D^{2}$ vary from 0 to 1 , but in all cases remain on the surface of the $V D C$ sphere, consistent with Eq. (2). concurrence (between the path states $\left|\mathbb{1}_{a}\right\rangle$ and $\left|\mathbb{1}_{b}\right\rangle$ and the polarization states $\left|s_{a}\right\rangle$ and $\left|s_{b}\right\rangle$ ) can be expressed in terms of the parameters $R$ and $\theta$,i.e.,

$$
V=\frac{2 R|\cos \theta|}{1+R^{2}}, \quad D=\left|\frac{1-R^{2}}{1+R^{2}}\right|, \quad C=\frac{2 R|\sin \theta|}{1+R^{2}} .
$$

Confirming results. For a given single-photon state, fringe visibility $V$ is achieved by registering the photon counts with an avalanche photodiode at the output of BS2 while continuously moving the translation stage. The particle distinguishability $D$ is obtained straightforwardly with photon counts by blocking first one and then the other of the two paths. Measurement of concurrence $C$ is realized by a tomographic analysis [28]. We measured $V, D$, and $C$ values for all seven single-photon states and the results are presented in Table I. They are also illustrated on the VDC sphere in Fig. 3, where the circled numbers correspond to the state numbers in Table I.

The SUM column in Table I reports $V^{2}+D^{2}+C^{2} \simeq 1$ for each one-photon state, states 1 to 7 . Those values represent confirmation of identity (2). As an example, one special "equal coherence" case of the three-way identity is achieved with $V=D=C$, indicating matching contributions of wave, particle, and entanglement. The corresponding one-photon self-entangled pure state is given as

$$
|\Psi\rangle=\sqrt{\frac{3+\sqrt{3}}{6}}\left|\mathbb{1}_{a}\right\rangle|h\rangle+\sqrt{\frac{3-\sqrt{3}}{12}}\left|\mathbb{1}_{b}\right\rangle(|h\rangle+|v\rangle) .
$$

The density matrix of the tomographically measured experimental state (7) is displayed in Fig. 4.

Concluding remarks. We observed with single-photon detection the QVE three-way identity $V^{2}+D^{2}+C^{2}=1$, for the quantum coherence possessed by single photons. At least

TABLE I. The measured values of visibility, distinguishability, and concurrence, where SUM refers to the identity combination $V^{2}+D^{2}+C^{2}$ and state 5 is an example supporting our remark that

\begin{tabular}{|c|c|c|c|c|c|}
\hline & $V$ & $D$ & $C$ & $V^{2}+D^{2}$ & $\mathrm{SUM} \simeq 1$ \\
\hline 1 & 0.992 & 0.009 & 0.003 & 0.985 & $0.985 \pm 0.014$ \\
\hline 2 & 0.719 & 0.680 & 0.012 & 0.980 & $0.980 \pm 0.054$ \\
\hline 3 & 0.068 & 0.994 & 0.008 & 0.992 & $0.992 \pm 0.060$ \\
\hline 4 & 0.048 & 0.708 & 0.703 & 0.503 & $0.998 \pm 0.084$ \\
\hline 5 & 0.058 & 0.011 & 0.991 & 0.004 & $0.986 \pm 0.040$ \\
\hline 6 & 0.720 & 0.011 & 0.691 & 0.518 & $0.996 \pm 0.070$ \\
\hline 7 & 0.587 & 0.568 & 0.570 & 0.667 & $0.992 \pm 0.070$ \\
\hline
\end{tabular}
even lossless pure states can have the traditional quantity of duality completely turned off-i.e., $V^{2}+D^{2} \simeq 0$. 


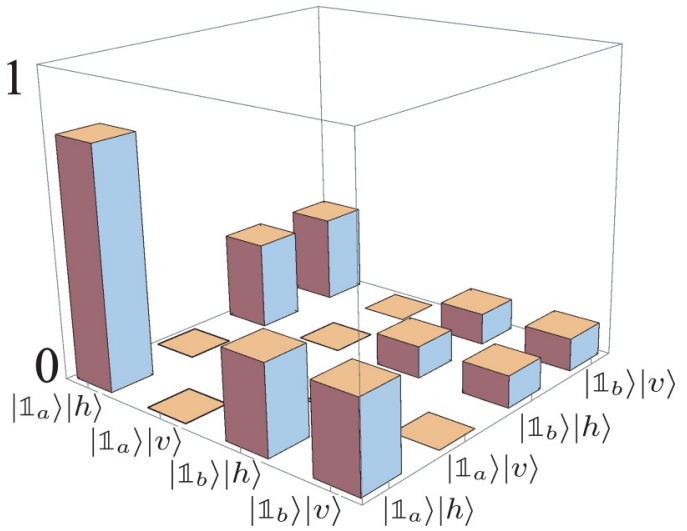

FIG. 4. Measured density matrix of state $|\Psi\rangle$ in Eq. (7).

since the 1980s, treatments of duality have been quantified in terms of $V$ and $D$ wave-particle analysis. This is shown to be incomplete. In the Young double-slit context the new identity (2) among $V, D$, and $C$ is seen to supplant it, establishing the important role for self-entanglement (nonseparability of degrees of freedom) in the self-interference of individual quantum particles.

As we understand duality, it refers to incompatible properties somehow found within the same particle. And it is particularly in the context of an individual particle that we find it surprising to discover that entanglement can rewrite duality's constraints. Multiparticle complementarities have been introduced, but, if several particles are joined in a common state, there might remain a way to imagine that the particle and wave characters can be shared between them. Thus they appear to us to redirect the duality discussion in a manner that we believe overlooks the issue.

Finally, there is relevance of these results even to the normally untestable one-particle principle of complementarity. Bohr's 1949 summary of his mandate (see Ref. [29]) reveals this point. As he said "...evidence obtained under different conditions cannot be comprehended within a single picture, but must be regarded as complementary in the sense that only the totality of the phenomena exhausts the possible information about the objects." Entanglement is a coherence that we believe was never considered by Bohr, and by taking account of single-particle self-entanglement our experiment supplies quantitative backup for his personal insights. Our results show experimentally that the repeatedly derived and employed wave-particle inequality $V^{2}+D^{2} \leqslant 1$ does not exhaust the possible information about a single quantum (recall the Wootters-Zurek analysis and a speculation by Knight in 1998 [30]). The completed QVE identity $V^{2}+D^{2}+C^{2}=1$, which adds single-particle entanglement, does so and says that by including self-entanglement all possible information of a single quantum object is exhausted in a two-slit experiment. In effect, we are reporting the experimental observation of the exhaustion of the coherences of single photons.

Acknowledgments. We are pleased to acknowledge conversations with colleagues G. S. Agarwal, I. Bialynicki-Birula, Paul Brumer, Steven Cundiff, F. De Zela, B.-G. Englert, A. Friberg, Peter Knight, Peter Milonni, Michael Raymer, L. Sanchez-Soto, W. P. Schleich, J. P. Torres, and W. H. Zurek. We acknowledge financial support from DARPA Grant No. D19AP00042 and from NSF Grants No. PHY-1203931, No. PHY-1505189, and No. INSPIRE PHY-1539859.
[1] See an early sketch of his wave-particle hypothesis in L. de Broglie, Waves and quanta, Nature (London) 112, 540 (1923); The later full presentation is Recherches sur la théorie des quanta, Ann. Phys. 10, 22 (1925). The English translation is by A. F. Kracklauer, On the theory of quanta, available from aflb.ensmp.fr/LDB-oeuvres/De_Broglie_Kracklauer.pdf.

[2] R. P. Feynman, The Feynman Lectures on Physics (AddisonWesley, Reading MA, 1963), Vol. 1, Sec. 37-2.

[3] Th. Young, An account of some cases of the production of colours, not hitherto described, Philos. Trans. R. Soc. London 92, 387 (1802).

[4] W. K. Wootters and W. H. Zurek, Complementarity in the double-slit experiment: Quantum nonseparability and a quantitative statement of Bohr's principle, Phys. Rev. D 19, 473 (1979).

[5] D. M. Greenberger and A. Yasin, Simultaneous wave and particle knowledge in a neutron interferometer, Phys. Lett. A 128, 391 (1988).

[6] G. Jaeger, A. Shimony, and L. Vaidman, Two interferometric complementarities, Phys. Rev. A 51, 54 (1995).

[7] B.-G. Englert, Fringe Visibility and Which-Way Information: An Inequality, Phys. Rev. Lett. 77, 2154 (1996).

[8] We keep in mind the lack of a firm relation between duality and complementarity. The former is de Broglie's simple flat statement about each single quantum object, and complementarity (notably not mentioned by Feynman) is Bohr's phrasing of the consequences of duality. Bohr's multiple rephrasings are well known; see Ref. [9].

[9] Bohr's multiply revised and delayed report of his first public pronouncement of complementarity to an international Physics Congress at Como in 1927 was published as The quantum postulate and the recent development of atomic theory, Nature (London) 121, 580 (1928); and Naturwissenschaften 16, 245 (1928); Reactions to complementarity in its earliest moments, including at the Como congress, are available in Max Jammer's histories: The Conceptual Development of Quantum Mechanics (McGraw-Hill, New York, 1966); and The Philosophy of Quantum Mechanics (Wiley, New York, 1974); Reflective analyses have never stopped: see D. Murdoch, Niels Bohr's Philosophy of Physics (Cambridge Univiversity, Cambridge, England, 1987); and A. Whitaker, Einstein, Bohr and the Quantum Dilemma, 2nd ed. (Cambridge University, Cambridge, England, 2006).

[10] S. Dürr, T. Nonn, and G. Rempe, Fringe Visibility and WhichWay Information in an Atom Interferometer, Phys. Rev. Lett. 81, 5705 (1998).

[11] Michal Kolar et al., Path and phase determination for an interfering photon with orbital angular momentum, Opt. Lett. 33, 67 (2008).

[12] P. D. D. Schwindt, P. G. Kwiat, and B. G. Englert, Quantitative wave-particle duality and nonerasing quantum erasure, Phys. Rev. A 60, 4285 (1999). 
[13] V. Jacques, E. Wu, F. Grosshans, F. Treussart, P. Grangier, A. Aspect, and J.-F. Roch, Delayed-Choice Test of Quantum Complementarity with Interfering Single Photons, Phys. Rev. Lett. 100, 220402 (2008).

[14] J. Tang, Y. Li, X. Xu, G. Xiang, C. Li, and G. Guo, Realization of quantum Wheeler's delayed-choice experiment, Nat. Photonics 6, 600 (2012).

[15] F. Kaiser, T. Coudreau, P. Milman, D. B. Ostrowsky, and S. Tanzilli, Entanglement-enabled delayed-choice experiment, Science 338, 637 (2012).

[16] A. G. Manning, R. I. Khakimov, R. G. Dall, and A. G. Truscott, Wheeler's delayed-choice gedanken experiment with a single atom, Nat. Phys. 11, 539 (2015).

[17] R. Menzel, D. Puhlmann, A. Heuer, and W. P. Schleich, Wave-particle dualism and complementarity unraveled by a different mode, Proc. Natl. Acad. Sci. USA 109, 9314 (2012).

[18] A. Norrman, K. Blomstedt, T. Setala, and A. T. Friberg, Complementarity and Polarization Modulation in Photon Interference, Phys. Rev. Lett. 119, 040401 (2017).

[19] E. Bagan, J. Calsamiglia, J. A. Bergou, and M. Hillery, Duality Games and Operational Duality Relations, Phys. Rev. Lett. 120, 050402 (2018).

[20] X.-F. Qian, A. N. Vamivakas, and J. H. Eberly, Entanglement limits duality and vice versa, Optica 5, 942 (2018).

[21] W. K. Wootters, Entanglement of Formation of an Arbitrary State of Two Qubits, Phys. Rev. Lett. 80, 2245 (1998).

[22] We note that a relation similar to Eq. (2) has been obtained in the context of two qubits: M. Jakob and J. A. Bergou, Quantitative complementarity relations in bipartite systems, arXiv:quantph/0302075, and later expanded in Opt. Commun. 283, 827 (2010), and also mentioned in a two-particle experimental proposal [23]. In our opinion, such complementarities, for more than one particle, are not relevant to de Broglie's duality.

[23] F. de Melo, S. P. Walborn, J. A. Bergou, and L. Davidovich, Quantum Nondemolition Circuit for Testing Bipartite Complementarity, Phys. Rev. Lett. 98, 250501 (2007).

[24] E. Schrödinger, Discussion of probability relations between separated systems, Math. Proc. Cambridge Philos. Soc. 31, 555 (1935).

[25] New results employing squeezed light are compatible with our conclusion that particle and wave features $(D$ and $V$ in our case) are insufficient to describe quantum duality. They do not conflict with our resolution given in identity (2). See J. Sperling, S. De, T. Nitsche, J. Tiedau, S. Barkhofen, B. Brecht, and C. Silberhorn, Wave-particle duality revisited: Neither wave nor particle, arXiv:1907.09836.

[26] T. T. Tran, K. Bray, M. J. Ford, M. Toth, and I. Aharonovich, Quantum emission from hexagonal boron nitride monolayers, Nat. Nanotechnol. 11, 37 (2016).

[27] K. Konthasinghe, C. Chakraborty, N. Mathur, L. Qiu, A. Mukherjee, G. D. Fuchs, and A. N. Vamivakas, Rabi oscillations and resonance fluorescence from a single hexagonal boron nitride quantum emitter, Optica 6, 542 (2019).

[28] D. F. V. James, P. G. Kwiat, W. J. Munro, and A. G. White, On the measurement of qubits, Phys. Rev. A 64, 052312 (2001).

[29] See comments in P. A. Schilpp, Editor, Albert Einstein: Philosopher-Scientist (Library of the Living Philosophers, Evanston, IL, 1949); and related commentary by D. Murdoch, Niels Bohr's Philosophy of Physics (Cambridge University, Cambridge, England, 1987); and A. Whitaker, Einstein, Bohr and the Quantum Dilemma, 2nd ed. (Cambridge University, Cambridge, England, 2006).

[30] P. L. Knight, Where the weirdness comes from, Nature (London) 395, 12 (1998). 